\title{
Learning Frequent Behaviors Patterns in Intelligent Environments for Attentiveness Level
}

\author{
Dalila Durães, ${ }^{1,3}$, Catarina Cardoso ${ }^{2}$,Javier Bajo ${ }^{1}$, Paulo Novais ${ }^{2}$ \\ ${ }^{1}$ Department of Artificial Intelligence, Technical University of Madrid, Madrid, Spain \\ d.alves@alumnos.upm.es, jbajo@fi.upm.es \\ ${ }^{2}$ Algoritmi Center, Minho University, Braga, Portugal \\ pg30462@alunos.uminho.pt, pjon@di.uminho.pt \\ ${ }^{3}$ CIICESI, ESTGF, Polytechnic Institute of Porto, Felgueiras, Portugal
}

\begin{abstract}
Nowadays, when it comes to achieving goals in business environments or educational environments, the performance successfully has an important role in performing a task. However, this performance can be affected by several factors. One of the most common is the lack of attention. The individual's attention in performing a task can be determinant for the final quality or even at the task's conclusion. In this paper is intended to design a solution that can reduce or even eliminate the lack of attention on performing a task. The idea consist on develop an architecture that capture the user behavior through the mouse and keyboard usage. Furthermore, the system will analyze how the devices are used.
\end{abstract}

Keywords: Attention, User Behavior, and Ambient Intelligent System

\section{Introduction}

Currently, one of the major problems related to work is the quantity of attention that people spend on performing a propose task. The level of attention of each person is increasingly affected by the evolution of Internet usage and introduction of social networks. These two factors had an high impact in attention because offers many information of general interest.

Research on attention involves nowadays many fields, including education, psychology, neuroscience, cognitive neuroscience and neuropsychology. For this reason, many different views and theories on attention can be found. One of the most frequent ones is the so-called "Attention Economics", which treats human attention as a scarce commodity or resource, which we must use wisely in order to attain our goals [1].

Attention is a very complex process through which one individual is able to continuously analyze a spectrum of stimuli and, in a sufficiently short amount of time, chose one to focus on [2]. In most of us, which can only focus on a very reduced group of stimuli at a time, this implies ignoring other perceivable stimuli and information.

adfa, p. 1, 2011.

(C) Springer-Verlag Berlin Heidelberg 2011 
The main goal of this paper is to propose an architecture to classify the level of attention of each user. This will provide the necessary information to the leadership, which help the user to improve his level of attention.

\section{Theoretical Foundations}

Nowadays the world is getting increasingly competitive and quality and quantity of the work presented is one of the decision factors in choosing a collaborator. It is no longer necessary to only perform, but, from that performance should result a product with quality, in time, at the lowest possible cost and with the minimum resources. It is essential that the factors that influence performance match the ideal values.

Attention is one of the factors that influence the performance of a human being when performing a task. If the attention of an individual is not at its best when performing the proposed task, its performance will be negatively affected, causing several problems.

In enterprise environments, if an employee has lack of attention in his work, that behavior can cause production delays. Consequently delays in delivery to costumers in extreme situations cause monetary losses. In some situations, such as, flight controllers or bus drivers, if the person is not $100 \%$ focused, an error can cause a tragic accident.

In educational environments, attention is considered a fundamental factor in the evolution and success of the student. If the student is not concentrating and paying attention to what is being taught, he will not capture information that is being provided and consequently the academic course will be compromised.

Attention is a resource that allows the human being to be focused on a situation and to be able to ignore non-priority information. As happens with performance, several factors can influence attention, like, stress, mental fatigue, anxiety, emotion, new environments, and human health. Besides these factors, the advancement of technology has been a real problem which has increased the lack of attention. With the emergence of the smartphone that provides new and varied information in real time and new ways of communication, people's attention is easily captured and the task that was meant to be done is left out $[3,4]$.

\subsection{Attention in Computer Science}

After being studied by psychology, cognitive psychology, and many other areas, attention is being studied by the field of computer science, because the number of people using new technologies to perform tasks is increasing and it is necessary to study how to increase the attention of people on their task while not being distracted by the amount of information that is provided by these same technologies [5].

Koch and Ullman are two authors who have studied attention in the computing world. They propose the "saliency map" [6]. "Saliency map" is a combination between different visual features that contribute to the selection of stimuli and a single type of topographic map. This proposal can offer the probability that each area of the 
visual field has to be captured. Later on, the proposal was developed by Laurent Itti. The first system had as input an image and the result was an initial image "saliency map". This was the starting point for many developed models not only for image but also for videos and more recently 3D. This is presented in Figure 3.

Another author who was interested in this branch is Matei Mancas [5-7]. According to Mancas, attention may have two approaches: the first approach is based on the two theories described above, saliency; the second approach is the visibility. In this model, it is assumed that an individual, when needing help to perform a task, looks for places where the information has good visibility. In this approach, the eye's movement gives an automatic output of the focus area. The Figure 4 presented an example of this second approach.

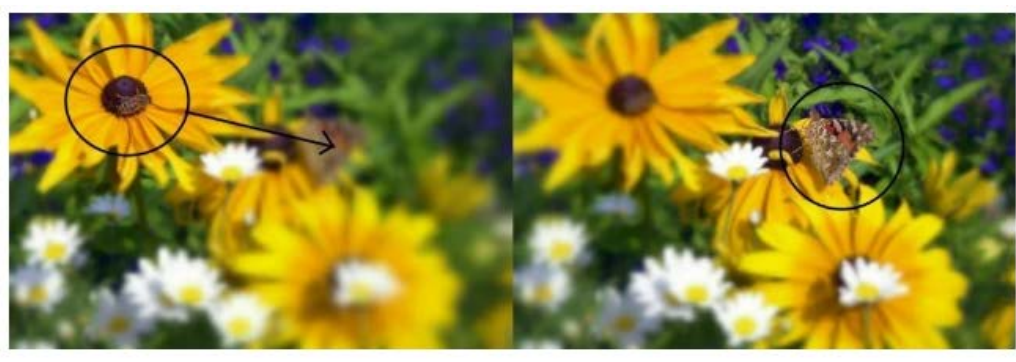

Fig. 1. Visibility output example [5]

In the figure 1, it is possible to visualize the same picture with the attention's focus targeted to two different areas. In the left, the observer's visual attention is directed to the flower, but in the right, the butterfly is the focus. These are two examples of image's outputs obtained with this approach.

\subsection{User behavior}

In the last years, task resolution using new technologies is increasing. Therefore, one of the biggest concerns is to find ways to make this use as efficient and effective as possible. The task resolution is not done similarly by all the users. Each person has its own behavior. This behavior can be derived from several factors, such as: biological characteristics of the user; task's characteristics; and environmental factors.

The recognition of these behaviors is already used in new technologies. For example, the recognition of users as a form of authentication on a device or software. This recognition is made by tracking of behavioral biometrics, such as, fingerprints, face recognition or iris recognition $[8,9]$.

According to Roman Yampolskiy and Venu Govindaraju [10], this behavioral biometrics can be classified in five categories based on information type:

- Behavioral Biometric based on the analysis of text extracts or drawings made by the user;

- Behavioral Biometric based on computer interaction; 
- Device usage, such as, mouse and keyboard. Devices that can capture the muscles' actions;

- Software interaction: strategy, and knowledge.

- Behavioral Biometric based in monitoring data coming from low level action in software, such as, access log, storage activity, and calls systems.

- Behavioral Biometric based on data from motor skills.

Behavioral Biometric based on strategy, skills in performing mentally demanding tasks. These monitored behaviors can be used to measure several variables that can influence the task development. There are already some studies that focus on recognition of some of these variables in the user behavior.

One of the variables is attention. To recognize the lack of attention on user behavior, different ways of capture and classify attention were discovered. One of the first ways to try to quantify the attention level was a questionnaire. This questionnaire is presented to the user. After the user answers the questionnaire, the study's author analyzes the answers and presents the conclusions. However, this approach is more qualitative and depends on the author's interpretation [11].

The approach more quantitative is the one that use biometrics behavioral. It is an approach more focused on the perception stimuli. Eye-tracking is one of the most used techniques. Using this technology when performing a task on a computer, it is possible to know the screen area where the eyes are directed and, consequently, where is the focus of attention. So it is possible to conclude if the user was with the visual attention directed to the screen area where the task is positioned [11, 12].

Finally, the mouse-tracking and keyboard-tracking are techniques used to measure and classify attention. These techniques have already been used to measure others variables, like, stress [13] and mental fatigue [14].

In the first phase, it was used statistic methods and the first conclusions were obtained. This data was analyzed in two ways:

- To find common behavior in groups of students;

- Individual data was analyzed for each student.

In the second phase, it was used a machine learning method to model the student's response to a stress event. There were used two classifiers. One to classify the all students' data, and other to classify data from an individual student.

This study concluded that stress affected the student's performance on an exam but raised some doubts about the reason for which some students can improve their performance even with stress.

\section{Proposed Design}

In Figure 2, it is presented the first version of the system's architecture, which is divided in three major parts. The lower-level is composed by the devices that generate the raw data (e.g. computers, smartphones). These devices store the raw data locally 
in SQLite databases, until it is synchronized with the web servers in the cloud, which happens at regular intervals (normally 5 minutes).

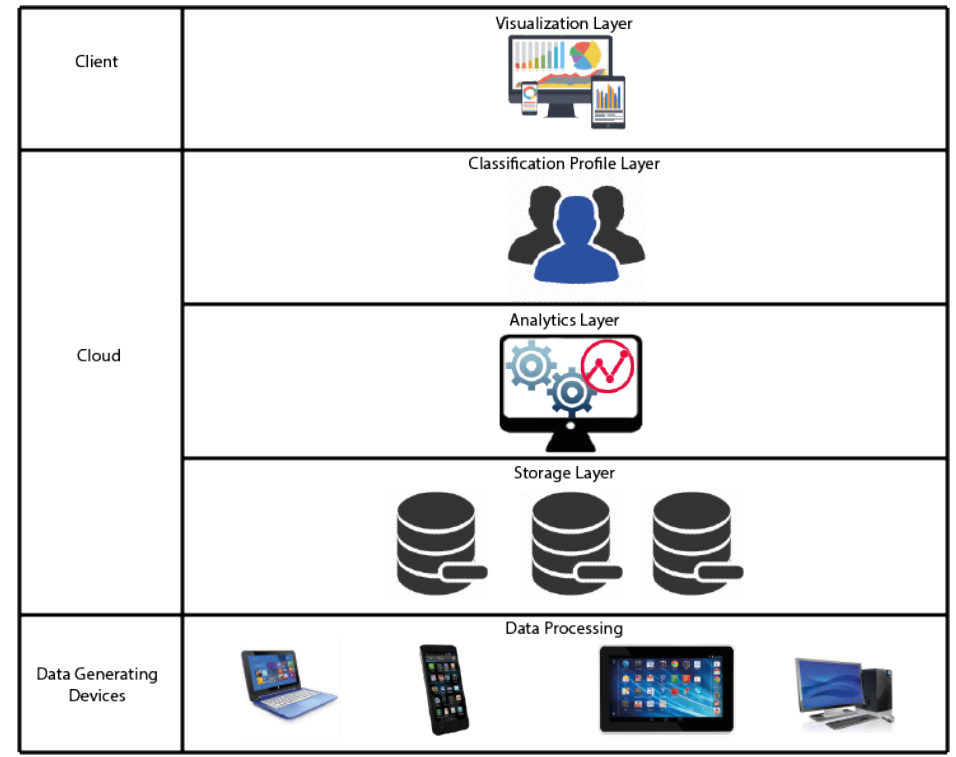

Fig. 2. Architecture of the system.

The cloud is composed of three layers: storage, analytics, and profile classification. In the storage layer the raw data received from the data generating devices is stored in a data store engine. The analytic layer provides powerful tools for performing analytics and statically analysis in real-time. The system calculates, at regular intervals, an estimation of the general level of performance and attention of each user.

The profile classification layer (Classifier) is where the indicators are interpreted. For example, interpreting data from the attentiveness indicators and build the meta-data, that will support decision-making. When the system has a sufficiently large dataset that allows making classifications with precision, it will classify the inputs received into different attention levels in real-time. This layer has access to the current and historical state of the group from a global perspective, but it can also refer to each user individually, creating each person learning profile [15]. The data is processed in Classifier (Classification Profile Layer). In Classifier, the analyzed data use the new information and compare with data from the profile saved.

Profile classification is also a very important aspect to have control of since it allows carrying out analyses within longer time frames. This information will be used by another sub-module, the affective adaptive agent, to provide relevant information to the platform and to the mentioned personalization module.

Finally, the Client layer is developed as a web app with intuitive and visual representation (diagrams and other graphical tools) of the attentiveness states of the group and each user, abstracting from the complexity of the data level where they are positioned. At this point, the system can start to be used by the people involved, especially 
the administrator, who can better adapt and personalize strategies. With a focus on individual and group performance and using real time analytics, the intuitive visual tools suggest and facilitate decision-making and student management.

\subsection{Classifier}

The system begins in the HTTP Service with the reception of a post request with three parameters, two dates and a set of task rules. Using the two dates, the Db Module collects the monitored data from a Mongo database.

The main goal of Classifier is to be able to classify the level of attention of each user. This will provide the necessary information to the leadership, which help the user to improve his level of attention.

The classifier is the software that classifies the level of attention. In the Figure 3, the classifier's architecture is presented. Three parts of equal value compose it: HTTP Service, DB Module and the Classifier.

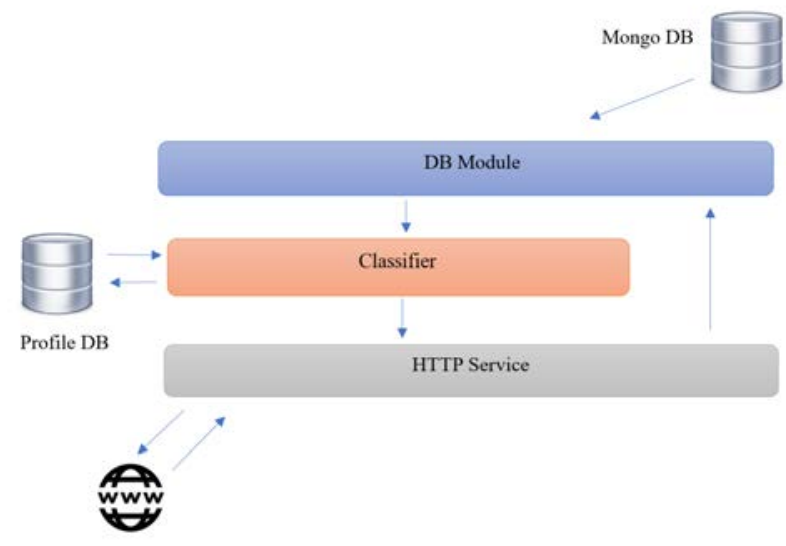

Fig. 3. Architecture of Classifier Module

The system begins in the HTTP Service with the reception of a post request with three parameters, two dates and a set of task rules. The two dates represent time interval for data collection. Using the two dates, the Db Module collects the monitored data from a Mongo database. The major goal of Db Module is to facilitate the collection of monitoring data. The module is composed by a MongoDB Drive, which makes the connection between a MongoDB, where the user information is stored, and the software system possible. The data of a specific date is collected from the MongoDB. The data collected is divided into three types: related to the task, interaction with the mouse and interaction with the keyboard.

The data is processed in Classifier. In Classifier, the analyzed data uses the new information and compares it with data from the saved profile. The data's analysis is done in two parts: user's attention classification and user's learning style definition. To classify it, it will be necessary to group the data per student. The task data from the 
new information is the most crucial information because from it derives most part of the attention level. To obtain the task results, the task rules received in the request are used to have a perception of how much time the user has spent on the applications related with the task rules.

As far as the mouse and keyboard results are concerned, their role is present on how the user interaction is occurring and help the coordinator/teacher understand if any student, who has the application active, is really working on it or not. To do that many values, such as mouse acceleration, mouse velocity, distance between clicks, duration of clicks, time between keys, key down time velocity are taken into account.

The data from the saved profile is stored in a MySQL database. This database store all the attention information previous collected and analyzed from the users.

To define the user's learning style, a mathematical method will be used. After the Classifier process is finished, the results of the user's attention level and the user's learning style will be sent back to the request's origin, with a response type JSON.

\section{$4 \quad$ Discussion and Future Directions}

Relatively to attention, it is an important theme because it is one of the factors that most influence a person's performance while performing a task. Therefore, it is a very studied theme by several areas, like, psychology, neuroscience and computer science. Understanding how attention varies and which situation the attention varies for each person, it is possible to act at the right moment and right time, to bring the user's attention level to the ideal value.

One way to quantify and classify the attention is the monitoring the user's behaviors when performing a task. Some approach, like, eye-tracking, neural activity caption or mouse-tracking/keyboard-tracking, can be used to monitor the behaviors. The project's approach will be the mouse-tracking/keyboard-tracking because it is a noninvasive and non-intrusive approach.

In previous work [15] the collected data were analyzed in two different ways. First, a general analysis was carried out with the aim of searching for group trends, i.e., behaviors common to a significantly large slice of user's. Secondly, an individual analysis is made, in order to compare the different moments.

One of the first results obtained were counts the number of interactions with the keyboard and the mouse in order to determine each data length user. In this step it was possible to verify the mouse and keyboard activity of each user which depends on the subjects that are applied [15].

The second results obtained were determine which applications are and are not work-related. In this sense it was necessary to measure the amount of time in each interval, that the user spent interacting with task-related applications. By default, applications that are not considered task-related are marked as "others" and count negatively towards the quantification of attention [15].

In the next step, it will start the system application's development to classify attention and define user's learning styles. At the end, it is expected to have all the proposed features presented in the first chapter developed on the most efficient way. 


\section{Acknowledgements}

This work has been supported by COMPETE: POCI-01-0145-FEDER-007043 and FCT - Fundação para a Ciência e Tecnologia within the Project Scope: UID/CEC/00319/2013.

\section{References}

1. Davenport, T.H., Beck, J.C. (2013). The Attention Economy: Understanding the New Currency of Business. Harvard Business Press.

2. Estes, W.K. (2014). Handbook of Learning and Cognitive Processes. Attention and Memory. Psychology Press, vol. 4.

3. Durães, D., Jiménez, A., Bajo, J., Novais, P. (2016). Monitoring Level Attention Approach in Learning Activities. Advances in Intelligent System and Computing, 478, 33-40. Doi: 10.1007/978-3-319-40165-2_4.

4. Pimenta, A., Carneiro, D., Novais, P., Neves, J. (2015). Detection of Distraction and Fatigue in Groups through the Analysis of International Patterns with Computers. Intelligent Distributed Computing VII, Springer International Publishing, 29-39.

5. Mancas, M. (2015). Attention in Computer Science - Part 1. News and insights from EAI community. [Cited: 31 December 2016.] http://blog.eai.eu/attention-in-computer-sciencepart-1/.

6. Koch, C., Ullman, S. (1987). Shifts in Selective Visual Attention: Towards the Underlying Neural Circuitry. Matters of Intelligence. Springer Netherlands, 115-141.

7. Mancas, M. (2015). Attention in Computer Science - Part 2. News and insights from EAI community. Cited: 31 December 2016. http://blog.eai.eu/attention-in-computer-sciencepart-2/.

8. Benevenuto, F., et al. (2009). Characterizing User Behavior in Online Social Networks. IMC '09 Proceedings of the 9th ACM SIGCOMM conference on Internet measurement conference, 49 - 62.

9. Risto, N., Gaillard, A. W. K., Mantysalo, S. (1978). Early Selective-Attention Effect on Evoked Potential Reinterpreted. . Acta Psychologica, 313-329.

10. Yampolskiy, R. V., Govindaraju, V. (2008). Behavioural Biometrics: a Survey and Classification .International Journal of Biometrics.

11. Mancas, M. (2015). How to Measure Attention? News and insights from EAI community Cited: 7 December 2017. http://blog.eai.eu/how-to-measure-attention/.

12. Duc, A., Hoang, B., Masud, P., Masud, H. (2008). Eye Movements as a Probe of Attention. Progress in Brain Research. 403-411.

13. Carneiro, D, et al. (2015). Using Mouse Dynamics to Assess Stress During Online Exams, 345-356.

14. Pimenta, A., et al. (2013). Monitoring Mental Fatigue through the Analysis of Keyboard and Mouse Interaction Patterns. Hybrid Artificial Intelligent Systems, 222-231.

15. Durães, D., Carneiro, D., Bajo, J., Novais, P. (2016). Supervising and Improving Attentiveness in Human Computer Interaction. Intelligent Environment 2016, 255- 264. Doi: 10.3233/978-1-61499-690-3-255. 